Sädhanā, Vol. 26, Part 5, October 2001, pp. 485-494. (C) Printed in India

\title{
Application of chaotic noise reduction techniques to chaotic data trained by ANN
}

\author{
C CHANDRA SHEKARA BHAT ${ }^{1}, \mathrm{M}^{\mathrm{R}} \mathrm{KAIMAL}^{2}$ and \\ T R RAMAMOHAN ${ }^{1}$ \\ ${ }^{1}$ Computational Materials Science, Unit-I, Regional Research Laboratory (CSIR) \\ Thiruvananthapuram 695 019, India \\ e-mail: csb@csrrltrd.ren.nic.in \\ 2 Department of Computer Science, University of Kerala, Kariavattom PO, \\ Thiruvananthapuram 695 581, India \\ e-mail: dcsku@md2.vsnl.net.in
}

MS received 27 March 2000

\begin{abstract}
We propose a novel method of combining artificial neural networks (ANNs) with chaotic noise reduction techniques that captures the metric and dynamic invariants of a chaotic time series, e.g. a time series obtained by iterating the logistic map in chaotic regimes. Our results indicate that while the feedforward neural network is capable of capturing the dynamical and metric invariants of chaotic time series within an error of about $25 \%$, ANNs along with chaotic noise reduction techniques, such as Hammel's method or the local projective method, can significantly improve these results. This further suggests that the effort on the ANN to train data corresponding to complex structures can be significantly reduced. This technique can be applied in areas like signal processing, data communication, image processing etc.
\end{abstract}

Keywords. Backpropagation algorithm; noise reduction; logistic map.

\section{Introduction}

Artificial neural networks (ANNs) are finding increasing use in many real problems. In particular, feedforward neural networks of interconnected nonlinear units are being used in many complex problems. The backpropagation algorithm (Rumelhart et al 1986) provides a mechanism for the calculation of the optimal weight matrix of multilayered networks composed of units with sigmoid activation functions, which exhibit nonlinear threshold characteristics.

Recently, many researchers have devoted much time and effort to representing chaotic phenomena using neural networks (Albano et al 1992; Ginsburg \& Horn 1992; Principe et al 1992; Deco \& Schurmann 1994; Nagayama \& Akamatsu 1994; Navone \& Ceccatto

A list of symbols is given at the end of the paper 
1995; Chandra Shekara Bhat \& Kaimal 1995, 1997). Nagayama \& Akamatsu (1994) have shown that a neural network can approximate chaotic behaviour in nonlinear dynamical systems. Two classes of chaotic systems were considered in their work, namely differential equation models as represented by the Lorenz equations, and systems of iterated maps, represented by the one-dimensional Logistic map and the two-dimensional Henon map. In the case of iterated maps their ANN was able to capture the Lyapunov exponent of the system. However, they were not able to capture the Lyapunov exponent in the case of the Lorenz equations. In their work they did not attempt to capture the metric invariants of the chaotic time series such as the correlation dimension, and in many situations ANNs fail to capture these metric invariants, which are characteristics of chaotic structures.

Deco \& Schurmann (1994) have considered recurrent networks that were able to capture the dynamic and metric invariants (correlation dimension and largest Lyapunov exponent) of chaotic time series. They have shown that dynamic invariants can be captured by feedforward neural networks, but recurrent learning is required to improve the dynamical modelling of a chaotic time series. As examples they have used the Henon map, the Logistic map and a real world chaotic time series that corresponds to the concentration of one of the species as a function of time in experiments in the Belousov-Zhabotinskii reaction in a well-stirred flow reactor.

In this paper, we report a new technique to improve the performance of feedforward ANNs for chaotic time series. Here, we report results of the application of two chaotic noise reduction techniques to data obtained from a feedforward ANN trained on chaotic data. The two techniques we consider are Hammel's method and the local projective method. We compare the performance of these two methods on the trained data obtained from the Logistic map.

Conventional approaches to noise reduction like the Weiner and the Kalman filters are not suitable for signals measured from chaotic systems, because the underlying dynamics of the chaotic system is not localized in either the time or the frequency domain. Methods of noise reduction are extensively used in communication, physical systems and experimental measurements. There are many methods for reducing noise in a chaotic time series.

Hammel (1990) has presented a method which can reduce the noise of a chaotic orbit on an attractor by more than ten orders of magnitude. This method is simple and fast. Its performance was analysed for several two-dimensional systems at moderate noise levels, including the Ikeda map. For this analysis the underlying maps were assumed to be known.

One of the major disadvantages of the backpropagation algorithm is the excessive training time taken for convergence. Using the method suggested here, the training time of the ANN for chaotic time series can be reduced significantly. The method consists of two steps. First, the data have to be trained using a feedforward ANN to a reasonably good error-free level. Second, a suitable chaotic noise reduction algorithm has to be applied to reduce dynamical noise in the trained data.

\section{Application of ANN to chaotic systems}

We have applied our technique to the logistic map given by $x_{n+1}=\mu x_{n}\left(1-x_{n}\right)$. A four layer ANN was constructed for training the data obtained from logistic map for various values of $\mu$ between 3.6 and 4.0. Forty sets of data were trained using the ANN and the data were reproduced within $25 \%$ error level. Further improvement was found to be difficult in most cases with reasonable effort. It was found that the correlation 
dimension for the trained sets were greater than that of the original data. On an average it was found that the correlation dimension of the test data was $30 \%$ to $40 \%$ greater than that of the original sets. This may imply that there is dynamical noise in the trained data. Therefore we chose two simple chaotic noise reduction techniques based on ideas developed by Hammel (1990) and Cawley \& Hsu (1992) to get a better representation of the original data.

\section{Noise reduction algorithm due to Hammel}

There are several ways by which a dynamical process, represented by $f$ can be obscured by noise. A sequence $\left\{x_{k}\right\}_{k=0}^{N}$ satisfying $x_{k+1}=f\left(x_{k}\right), 0 \leq k \leq N-1$ will be called a true orbit. If a noisy orbit $\left\{p_{k}\right\}_{k=0}^{N}$ is generated by $p_{k}=x_{k}+\eta_{k}$ for small $\left\|\eta_{k}\right\|<\delta$, this will be termed additive noise. This method of noise generation can be imagined to occur when a true process is obscured by errors in measurement. The obvious goal is to reduce $\left\|\eta_{k}\right\|$. A second way by which the dynamics can be obscured is associated with the shadowing problem. As the noisy orbit $\left\{p_{k}\right\}_{k=0}^{N}$ is generated by the process $f$, errors are made upon each iteration of the process. The resultant points of the orbit then satisfy

$$
\left\|p_{k+1}-f\left(p_{k}\right)\right\|<\delta, \quad 0 \leq k \leq N-1,
$$

for an appropriately small $\delta$. This type of noise is called dynamic noise.

The noise reduction problem is to find a less noisy orbit near $\left\{p_{k}\right\}_{k=0}^{N}$. At one limit of nearness lies the old original noisy $\left\{p_{k}\right\}_{k=0}^{N}$; at the other extreme, one can seek a noise free true orbit $\left\{x_{k}\right\}_{k=0}^{N}$ which lies somewhat near $\left\{p_{k}\right\}_{k=0}^{N}$. This latter alternative is associated with the shadowing property. The orbit of $x_{0}$ is said to $\epsilon$-shadow $\left\{p_{k}\right\}_{k=0}^{N}$ if

$$
\left|f^{k}\left(x_{0}\right)-p_{k}\right|<\epsilon, \quad 0 \leq k \leq N .
$$

\section{The algorithm for noise reduction in the case of the logistic map}

The basic equation considered is

$$
\phi_{k}=\frac{d f}{d x} \phi_{k-1}+\pi_{k}, \quad k=1,2, \ldots, N,
$$

where

$$
\begin{aligned}
\phi_{k} & =x_{k}-p_{k}, \\
\pi_{k} & =f\left(p_{k-1}\right)-p_{k} .
\end{aligned}
$$

The algorithm applied to (3) is:

Step 1: Set $\phi_{N}=0$.

Step 2: If $\left|\frac{d f}{d x}\right|>1$ then iterate (3) backward, i.e. calculate

$$
\phi_{k-1}=\left(\phi_{k}-\pi_{k}\right) /\left.\frac{d f}{d x}\right|_{p_{k-1}} .
$$


Step 3: Else iterate (3) forward, i.e. calculate

$$
\phi_{k}=\left.\phi_{k-1} \frac{d f}{d x}\right|_{p_{k-1}}+\pi_{k}
$$

Step 4: Repeat steps 2 and 3 until the error is less than $\epsilon$, a small number.

\section{Results}

We present the results of our calculations on a few selected data sets obtained by iterating the logistic map at different parameter values. As can be seen from figures 1-2, the chaotic noise reduction technique reproduces the original curve to a great extent and as shown in table 1 , the correlation dimension of the ANN trained data is usually about $30 \%$ to $40 \%$ higher than that of the original data. However, upon application of the noise reduction algorithm, the corrected values approach the values corresponding to the original data quite significantly.

\section{Local projective noise reduction}

In order to compare the results of noise reduction by Hammel's method, we have also used another technique, viz. local projective noise reduction (Kantz and Schreiber, 1997) and tried to reduce the noise in ANN trained data of the Logistic map. Here unlike the Hammel's method the underlying dynamics of the system need not be known. A brief description of the method is given below.

\subsection{Brief description of the method}

Using local linear filters we can approximate the dynamical equations underlying a

deterministic signal. Here, we will assume that the map $\hat{F}$ (or the differential equation) is a smooth function of the coordinates, that is, it is atleast piecewise differentiable. Then we can linearise $\hat{F}$ locally in the vicinity of each point $x_{n}$ :

$$
\begin{aligned}
\hat{F}(x) & =\hat{F}\left(x_{n}\right)+J_{0}\left(x-x_{n}\right)+O\left(\left\|x-x_{n}\right\|^{2}\right) \\
& \approx J_{n} x+b_{n} .
\end{aligned}
$$

The matrix $J_{n}$ is the Jacobian of $\hat{F}$ in $x_{n}$.

We have noted above that if we have the description of the dynamics in an explicit, forward-in-time form we can perform nonlinear noise reduction only with the help of a separate trajectory adjustment step. This is necessary because of the sensitive dependence on initial conditions in chaotic systems. The basic idea here is to correct each delay vector $\beta_{n}$ individually by solving a minimisation problem which renders the corrected vector more consistent with the dynamics.

We express the above linearised relationship in an implicit way using delay coordinates. An $m-1$ dimensional map $x_{n}=F\left(x_{n-m+1}, \ldots, x_{n-1}\right)$ can be written implicitly as $\tilde{F}\left(x_{n-m+1}, \ldots, x_{n}\right) \equiv \tilde{F}\left(x_{n}\right)=0$, or linearised as

$$
a^{(n)} \cdot R\left(x_{n}-\bar{x}^{(n)}\right)=0+O\left(\left\|x_{n}-\bar{x}^{(n)}\right\|^{2}\right) .
$$



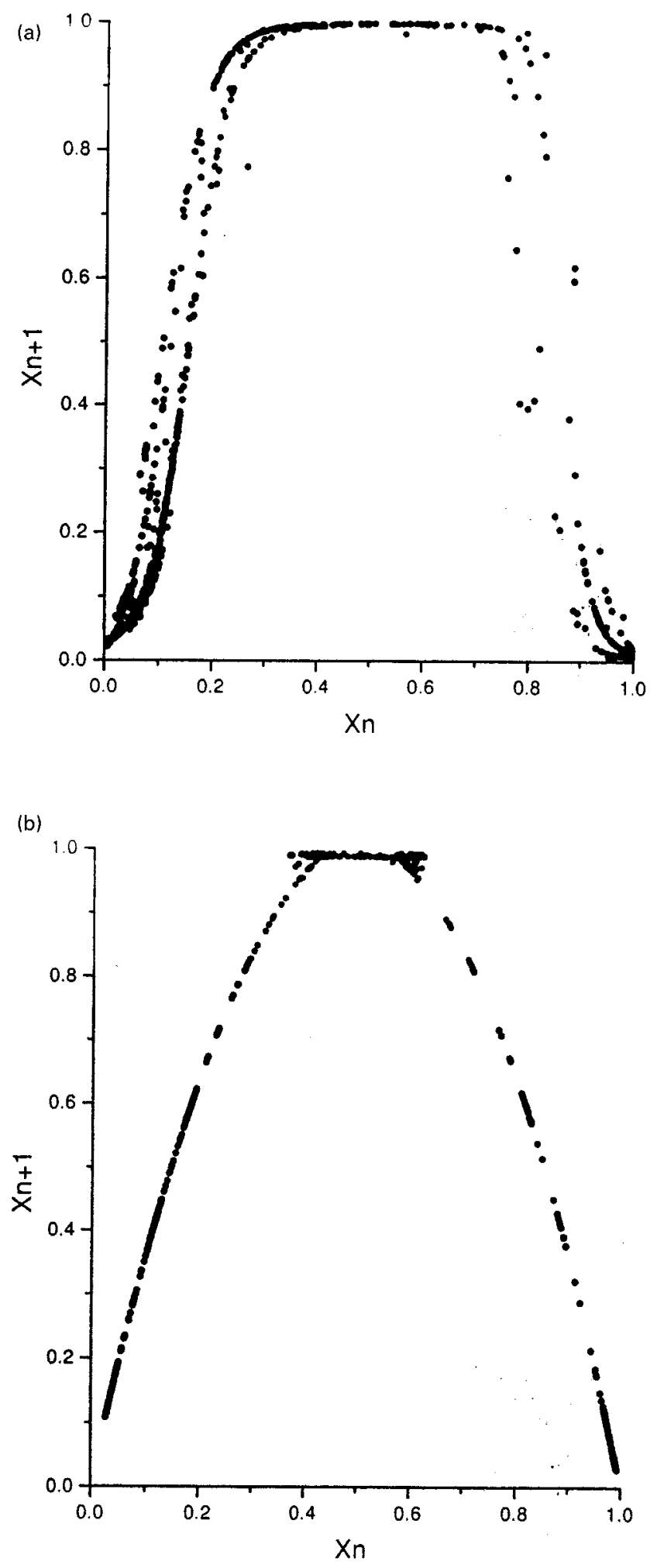

Figure 1. Return map of the (a) trained, and (b) noise-reduced logistic map for $\mu=3.99$. 

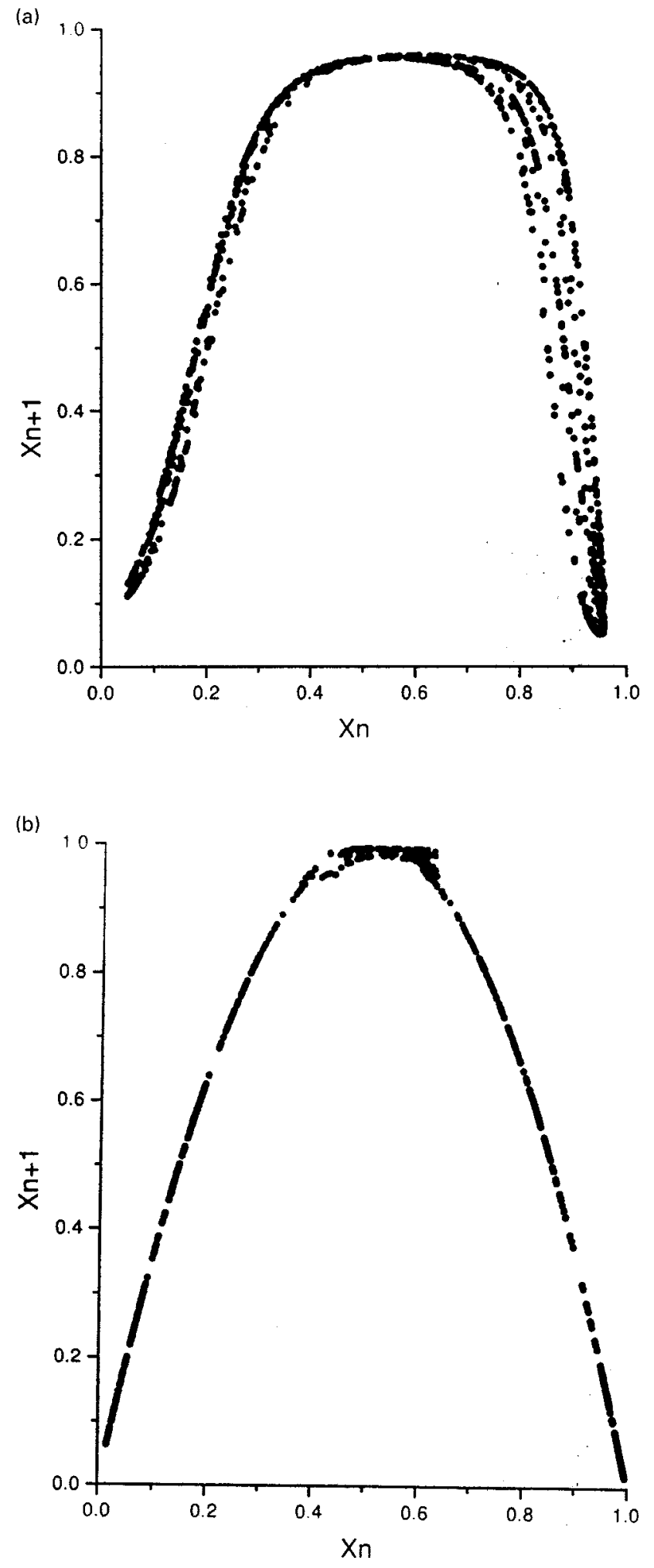

Figure 2. Return map of the (a) trained, and (b) noise-reduced logistic map for $\mu=4.00$. 
Table 1. Lyapunov exponent and correlation dimension of original, trained and noise-reduced data for Hammel's technique.

\begin{tabular}{lccc}
\hline$\mu$ & & Lyapunov exponent & Correlation dimension \\
\hline 3.70 & Original & $0.520 \pm 0.029$ & $0.991 \pm 0.028$ \\
& Trained & $0.511 \pm 0.030$ & $1.501 \pm 0.036$ \\
3.71 & Noise red. & $0.656 \pm 0.050$ & $1.025 \pm 0.149$ \\
& Original & $0.525 \pm 0.031$ & $0.938 \pm 0.061$ \\
& Trained & $0.443 \pm 0.086$ & $1.425 \pm 0.116$ \\
3.80 & Noise red. & $0.598 \pm 0.098$ & $0.987 \pm 0.066$ \\
& Original & $0.625 \pm 0.035$ & $0.953 \pm 0.018$ \\
& Trained & $0.596 \pm 0.066$ & $1.699 \pm 0.052$ \\
3.82 & Noise red. & $0.420 \pm 0.048$ & $1.060 \pm 0.054$ \\
& Original & $0.658 \pm 0.026$ & $0.969 \pm 0.045$ \\
& Trained & $0.728 \pm 0.085$ & $1.576 \pm 0.182$ \\
3.88 & Noise red. & $0.680 \pm 0.081$ & $1.036 \pm 0.078$ \\
& Original & $0.658 \pm 0.038$ & $0.932 \pm 0.023$ \\
& Trained & $0.559 \pm 0.079$ & $1.382 \pm 0.175$ \\
3.92 & Noise red. & $0.646 \pm 0.097$ & $0.967 \pm 0.086$ \\
& Original & $0.757 \pm 0.035$ & $0.962 \pm 0.072$ \\
& Trained & $0.498 \pm 0.033$ & $1.349 \pm 0.087$ \\
3.99 & Noise red. & $0.593 \pm 0.092$ & $1.008 \pm 0.083$ \\
& Original & $0.908 \pm 0.038$ & $0.982 \pm 0.057$ \\
& Trained & $0.495 \pm 0.142$ & $1.154 \pm 0.127$ \\
& Noise red. & $0.996 \pm 0.070$ & $0.996 \pm 0.070$ \\
\hline \multirow{3}{*}{30} & &
\end{tabular}

Here,

$$
\bar{x}^{(n)}=\left|\mathcal{U}_{n}\right|^{-1} \sum_{n^{\prime} \in \mathcal{U}_{n}} x_{n^{\prime}},
$$

is the centre of mass of the delay vectors in a small neighbourhood $\mathcal{U}_{n}$ of $x_{n}$. We introduce a diagonal weight matrix $R$ so that the noise reduction is focused on the most stable middle coordinates of the delay vectors. This can be done by choosing $R_{11}$ and $R_{m m}$ large and all other diagonal entries $R_{i i}=1$. For $R=1$ we would obtain orthogonal projections. The above relationship will not be valid exactly for a noisy sequence $s_{n}$, but to a limited extent error related to the noise:

$$
a^{(n)} \cdot R\left(\beta_{n}-\bar{\beta}^{(n)}\right)=\eta_{n}
$$

This means that there is one direction in phase space, $a^{(n)}$, into which the attractor does not extend to linear approximation. In the following we will suppress the notation $(n)$ but keep in mind that this linear equation is valid only locally and that the direction $a^{(n)}$, as well as the centre of mass $\bar{\beta}^{(n)}$, depends on the position in phase space.

We use a delay reconstruction in $m$ dimensions while the dynamics confine the trajectories to a lower-dimensional manifold of dimension $m_{0}$. Here, we can find up to $Q=m-m_{0}$ mutuallly independent sub-spaces $a^{q}, q=1, \ldots, Q$ fulfilling (8) and (9). We call the linear space spanned by these $Q$ vectors the nullspace at point $x_{n}$. Since the noise-free attractor does not extend to this space, the component of $\beta_{n}$ that we find in it must be due to noise. The locally projective noise reduction algorithm tries to identify this nullspace and then removes the corresponding component of $\beta_{n}$. 
We have to find $Q$ orthonormal vectors $a^{q}$ such that the local projection onto these vectors is minimal. If we use the notation $z_{n}=R\left(\beta_{n}-\bar{\beta}\right)$, the projection of $z_{n}$ onto the nullspace is $\sum_{q=1}^{Q} a^{q} \cdot\left(a^{q} \cdot z_{n}\right)$ and we require $\sum_{n^{\prime} \in \mathcal{U}_{n}}\left[\sum_{q=1}^{Q} a^{q} \cdot\left(a^{q} \cdot z_{n} \alpha\right)\right]^{2}$ to be minimal for the correct choice of the set of $a^{q}$. If we introduce the constraint that the $a^{q}$ have unit length by means of Lagrange multipliers $\lambda^{q}$ and use the fact that the $a^{q}$ are orthogonal, $a^{q} \cdot a^{q^{\prime}}=0, q \neq q^{\prime}$, we have to minimise the Lagrangian,

$$
L=\sum_{n^{\prime} \in \mathcal{U}_{n}}\left[\sum_{q=1}^{Q} a^{q} \cdot\left(a^{q} \cdot z_{n^{\prime}}\right)\right]^{2}-\sum_{q=1}^{Q} \lambda^{q}\left(a^{q} \cdot a^{q}-1\right),
$$

with respect to $a^{q}$ and $\lambda^{q}$. This can be done seperately for each $q$ and yields

$$
C a^{q}-\lambda^{q} a^{q}=0, \quad q=1, \ldots, Q,
$$

where $C$ is the $m X m$ covariance matrix of the vectors $z_{n^{\prime}}$ within the neighbourhood $\mathcal{U}_{n}$,

$$
C_{i j}=\sum_{n^{\prime} \in \mathcal{U}_{n}}\left(z_{n}^{\prime}\right)_{i}\left(z_{n}^{\prime}\right)_{j}
$$

The solutions of (11) are nothing but the orthogonal eigenvectors $a^{q}$ and eigenvalues $\lambda^{q}$ of $C$. The global minimum is given by the eigenvectors to the $Q$ smallest eigenvalues. The noise component of the vector $z_{n}$ is thus removed by replacing it by

$$
\hat{z}_{n}=z_{n}-\sum_{q=1}^{Q} a^{q} \cdot\left(a^{q} \cdot z_{n}\right) .
$$

Finally, we write the result in terms of the original delay vectors $\beta_{n}$ :

$$
\hat{\beta}_{n}=\beta_{n}-\Delta \beta_{n}=\beta_{n}-R^{-1} \sum_{q=1}^{Q} a^{q} \cdot\left[a^{q} \cdot R\left(\beta_{n}-\bar{\beta}\right)\right] .
$$

\section{Results}

We have applied the above technique to data obtained from the ANN trained logistic map. Compared to Hammel's method, results obtained from the local projective noise reduction method are not very encouraging, since the error reduction is not significant. However, in the absence of the underlying dynamics of the system one can use this method. The Lyapunov exponent and Correlation dimension for the noise reduced data have been calculated and are given in table 2 .

\section{Conclusions}

We have proposed a novel method of combining ANN's with chaotic noise reduction technique that captures the metric and dynamic invariants of a chaotic time series. We have also tried to combine the local projective method with ANN training to reduce the noise contained in the trained data. We have compared these two results and concluded that Hammel's noise reduction method yields better results than the local projective 
Table 2. Lyapunov exponent and correlation dimension of original, trained and noise-reduced data for local projective method.

\begin{tabular}{lccc}
\hline$\mu$ & & Lyapunov exponent & Correlation dimension \\
\hline 3.90 & Original & $0.718 \pm 0.031$ & $0.946 \pm 0.025$ \\
& Trained & $0.537 \pm 0.078$ & $1.144 \pm 0.183$ \\
& Noise red. & $0.500 \pm 0.076$ & $0.940 \pm 0.072$ \\
3.91 & Original & $0.682 \pm 0.042$ & $0.910 \pm 0.630$ \\
& Trained & $0.598 \pm 0.046$ & $1.345 \pm 0.095$ \\
& Noise red. & $0.381 \pm 0.081$ & $1.049 \pm 0.063$ \\
3.93 & Original & $0.788 \pm 0.034$ & $1.006 \pm 0.045$ \\
& Trained & $0.592 \pm 0.035$ & $1.340 \pm 0.152$ \\
& Noise red. & $0.376 \pm 0.071$ & $0.974 \pm 0.088$ \\
3.98 & Original & $0.877 \pm 0.034$ & $0.968 \pm 0.060$ \\
& Trained & $0.708 \pm 0.106$ & $1.365 \pm 0.079$ \\
& Noise red. & $0.533 \pm 0.076$ & $1.069 \pm 0.099$ \\
3.99 & Original & $0.908 \pm 0.038$ & $0.982 \pm 0.057$ \\
& Trained & $0.495 \pm 0.142$ & $1.154 \pm 0.127$ \\
& Noise red. & $0.389 \pm 0.083$ & $0.945 \pm 0.107$ \\
4.00 & Original & $0.960 \pm 0.039$ & $0.998 \pm 0.055$ \\
& Trained & $0.928 \pm 0.044$ & $1.366 \pm 0.123$ \\
& Noise red. & $0.635 \pm 0.083$ & $0.940 \pm 0.107$ \\
\hline
\end{tabular}

method. However we note here that the Hammel's technique needs knowledge of the underlying map whereas the local projective method does not require this. This technique of postprocessing the data obtained from a moderately well-trained ANN with chaotic noise reduction techniques seems to capture both dynamic and metric invariants of chaotic time series with relatively little effort and this may be of considerable significance in analysing such data.

We wish to thank Drs G Vijay Nair and B C Pai for their constant encouragement and support.

\section{List of symbols}

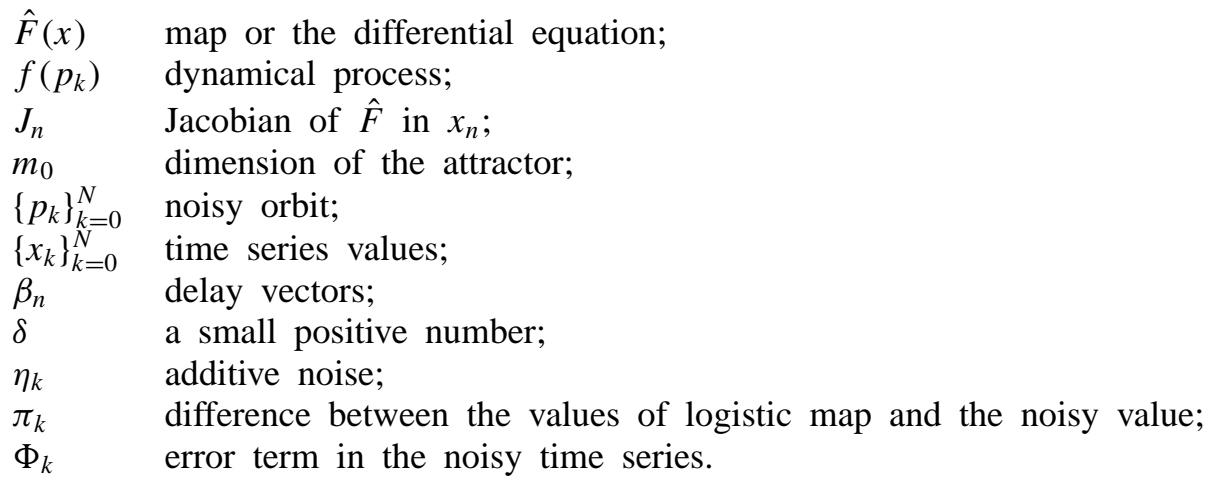




\section{References}

Albano A M, Passamante A, Hediger T, Farrell M E 1992 Using neural nets to look for chaos. Physica D58: 1-9

Cawley R and Hsu G H 1992 Local projection method for noise reduction in chaotic maps and flows. Phys. Rev. A46: 3057-3066

Chandra Shekara Bhat C, Kaimal M R 1995 Approximation of chaotic behavior using artificial neural networks. Proceedings of the Third International Seminar on Intelligent Robotic Systems, Bangalore

Chandra Shekara Bhat C, Kaimal M R 1997 Identification and reproduction of bifurcation diagram of logistic map using ANN. Proceedings of the National Conference on Neuro-Fuzzy Systems (New Delhi: Narosa)

Deco G, Schurmann B 1994 Neural learning of chaotic system behavior. IEICE Trans. Fundamentals E77-A: 1840-1845

Ginsburg I, Horn D 1992 Learning the rule of time series. Int. J. Neural Syst. 3: 167-177

Hammel S M 1990 A noise reduction method for chaotic systems. Phys. Lett. A148: 421-429

Kantz H, Schreiber T 1997 Nonlinear time series analysis (Cambridge: University Press)

Nagayama I, Akamatsu N 1994 Approximation of chaotic behavior by using neural network. IEICE Trans. Inf. Syst. E77-D: 450-458

Navone H D, Ceccatto H A 1995 Learning chaotic dynamics by neural networks. Chaos, Solitons Fractals 6: 383-387

Principe J, Rathie A, Kuo J 1992 Prediction of chaotic time series with neural networks and the issue of dynamical modelling. Int. J. Bifurcation Chaos 2: 989-996

Rumelhart D E, Hinton G E, Williams R J 1986 Learning internal representations by error propagation. Parallel distributed processing: Explorations in the microstructures of cognition (eds) D E Rumelhart, J L McClelland, (Cambridge, MA: MIT Press) 1: 318-362 